\title{
A randomized evaluation of simulation training on performance of vascular anastomosis on a high-fidelity in vivo model: The role of deliberate practice
}

\author{
Joel Price, MD, MPH, ${ }^{a}$ Viren Naik, MD, Med, ${ }^{\mathrm{b}}$ Munir Boodhwani, MD, MMSc, ${ }^{\mathrm{a}}$ Tim Brandys, MD, Med, ${ }^{\mathrm{c}}$ \\ Paul Hendry, MD, ${ }^{\mathrm{a}}$ and Buu-Khanh Lam, MD, MPH ${ }^{\mathrm{a}}$
}

\begin{abstract}
Objectives: There is mounting evidence supporting the benefit of surgical skills practice in a simulated environment. However, the use of simulation in cardiac surgical training has been limited. The purpose of the current trial was to examine the effect of independent and deliberate simulator practice, during nonclinical time, on the performance of an end-to-side microvascular anastomosis in an in vivo model.
\end{abstract}

\begin{abstract}
Methods: This single-blinded, randomized controlled trial received institutional review board approval. Thirtynine first- and second-year surgical trainees were randomized to an expert-guided tutorial on a procedural trainer or to the expert-guided tutorial combined with self-directed practice on the same procedural trainer. Selfdirected practice consisted of 10 anastomoses performed on the procedural trainer: a low-fidelity, commercially available bench model using 4-mm polytetrafluoroethylene graft as simulated blood vessel. Two weeks after the tutorial, subjects performed an end-to-side anastomosis in a live porcine model, under realistic operating room conditions. Assessment of outcomes was performed by 2 blinded, expert observers, uings validated measurements of technical skill. The primary outcome was the score on the Objective Structured Assessment of Technical Skill (OSATS) scale. Secondary outcomes included an anastomosis-specific end-product evaluation and time to completion. Statistical analysis was conducted using nonparametric, univariate techniques.
\end{abstract}

Results: Compared with residents who received expert-guided simulator training alone, those who in addition practiced on a simulator independently after hours scored significantly higher on the OSATS scale ( $23.7 \pm 4.7 \mathrm{vs}$ $18.5 \pm 3.9, P=.003)$. Residents who practiced independently also scored significantly higher on the endproduct evaluation $(11.4 \pm 3.2$ vs $8.9 \pm 2.1, P=.02)$ and performed the anastomosis significantly faster (777 seconds vs 977 seconds, $P=.04$ ). Interrater reliability was high between the expert observers (intraclass correlation coefficient $=0.8$ ).

Conclusions: Residents who had the opportunity for self-directed simulator practice performed an end-to-side anastomosis more adeptly, more quickly, and with a higher quality end product. The results of this randomized trial suggest that independent training on a procedural trainer did transfer to improved performance in an operating room environment. Simulator training should be incorporated into cardiovascular surgical curricula and residents should have access to this modality for independent after-hours practice to improve operating room performance. (J Thorac Cardiovasc Surg 2011;142:496-503)

The current method of surgical training is predicated on the apprenticeship model introduced by Halsted at Johns Hopkins over a century ago. ${ }^{1}$ Teaching of technical skills takes

From the Division of Cardiac Surgery, ${ }^{\text {a }}$ University of Ottawa Heart Institute, and the Department of Anesthesiology ${ }^{b}$ and Division of Vascular Surgery, ${ }^{c}$ The Ottawa Hospital, Ottawa, Ontario, Canada.

Supported by a grant from the University of Ottawa's Academy for Innovation in Medical Education-AIME Education Grant. Supplies donated by W.L. Gore \& Associates, Inc. Flagstaff, Ariz, and Covidien, USA.

Disclosures: Authors have nothing to disclose with regard to commercial support.

Read at the 91st Annual Meeting of The American Association for Thoracic Surgery, Philadelphia, Pennsylvania, May 7-11, 2011.

Received for publication April 15, 2011; accepted for publication May 17, 2011; available ahead of print July 11, 2011

Address for reprints: Joel Price, MD, MPH, Division of Cardiac Surgery, University of Ottawa Heart Institute, 40 Ruskin St, Ottawa, Ontario, Canada K1Y 4W7 (E-mail: dr.joel.price@gmail.com).

0022-5223/\$36.00

Copyright (c) 2011 by The American Association for Thoracic Surgery doi:10.1016/j.jtcvs.2011.05.015 place primarily in the operating room on live patients. There are a number of threats to this style of surgical education with the changing practice patterns of the new millennium. These include work-hour restrictions for medical professionals, an increasingly litigious medical environment, and increasing complexity of cases at surgical teaching centers. These factors have contributed to fewer opportunities to learn surgical procedures in the operating room on live patients. Furthermore, the high-stress environment of the operating room may not be the ideal environment for new skill acquisition. These challenges have stimulated the pursuit of novel techniques for teaching trainees outside the operating room. Simulation provides the learner with an opportunity for repetitive and deliberate practice of procedural skills in a low-consequence environment. The utility of simulation in surgical training is gaining widespread recognition; there is increasing literature demonstrating the 


\section{Abbreviations and Acronyms \\ OSATS $=$ Objective Structured Assessment of Technical Skill \\ PGY = postgraduate year}

and principles involved in performing an end-to-side anastomosis. The subjects were then given the opportunity to practice on the procedural trainers. Expert tutors were present to provide instruction and guidance to the participants. Each student performed 3 anastomoses, observed and guided by an expert. A fourth anastomosis was performed without coaching and recorded for scoring as a pretest. All students randomized to the expertguided tutorial alone were instructed not to practice the techniques outside of the laboratory.

Subjects randomized to receive the combination of expert-guided tutorial and self-directed practice on the procedural trainer received the identical training and pretest to that described for the first group. In addition, subjects randomized to this group were given a model, surgical instruments, suture material, and a $\log$ book. Self-directed practice involved performing 10 anastomoses that were done at the subject's convenience at some point during the 2 weeks after the training session. Subjects logged the date of each anastomosis they performed independently.

\section{Outcome Measures}

The primary end point was the mean Objective Structured Assessment of Technical Skill (OSATS) score for the anastomosis in the in vivo porcine carotid artery model. Secondary outcomes were time to completion and the end-product evaluation. The in vivo test occurred 2 weeks after the initial training session. The intention of the animal model was to create a highfidelity simulation in a realistic operating room environment. Several measures were used to ensure adequate face and content validity. To approximate pressures of the operating room environment, the trainee adhered to full sterile procedure and operating room protocol. In addition, subjects were made aware that they were being timed and graded. Juvenile Yorkshire swine $(35-50 \mathrm{~kg})$ underwent general anesthesia, mechanical ventilation, and cardiopulmonary monitoring similar to a human operating theater. The carotid arteries were exposed and clamped. Polytetrafluoroethylene tubing, 4 $\mathrm{mm}$ in diameter, served as a conduit for the anastomosis. The anastomosis was performed using a single, running 6-0 polypropylene suture.

All anastomoses were video recorded and subsequently graded by 2 expert observers. The graders were 2 expert cardiac surgeons who were blinded to the randomization status of the subjects. The observers were trained with sample performances in the appropriate use of anchored grading scales. The video recording was limited to the subject's hands and the model only, to avoid any possibility of identifying the subject.

Objective scoring of technical proficiency was conducted using 3 validated methods. The OSATS is a global rating scale developed at the University of Toronto. It has been extensively validated ${ }^{13-15}$ and used. The OSATS global rating scale is constructed of 7 categories marked on a Likert scale from 1 to 5, tied with descriptive anchors, giving a possible total of 35 marks (Appendix 1). A grade from 1 to 5 was assigned for each of the 7 fields in the modified OSATS global rating scale. A total score out of 35 will be assigned to each subject. The OSATS has been used and validated to score performance of vascular anastomoses in previous studies. ${ }^{2,10,11}$ Time to completion of a task has been widely applied for assessment of surgical skill. ${ }^{2,6,9,12}$ It has been suggested that while speed itself is not the goal of a surgeon, with increasing experience speed represents increasing economy of motion rather than haste. ${ }^{2}$ Subjects began the task from a standardized position. The time to tying of the final knot was recorded. The final outcome measure was the end-product evaluation. The end product was assessed on a 4-parameter global rating scale by blinded observers. ${ }^{12}$ Four parameters-suture spacing, suture eversion, quality of the heel of the anastomosis, and the quality of the anastomotic toewere graded on a 5-point Likert scale, with the total mark out of 20 (Appendix 2).

\section{STATISTICAL ANALYSIS}

A sample size calculation was performed using SAS version 9.1.3 statistical software (SAS Institute, Inc, Cary, 


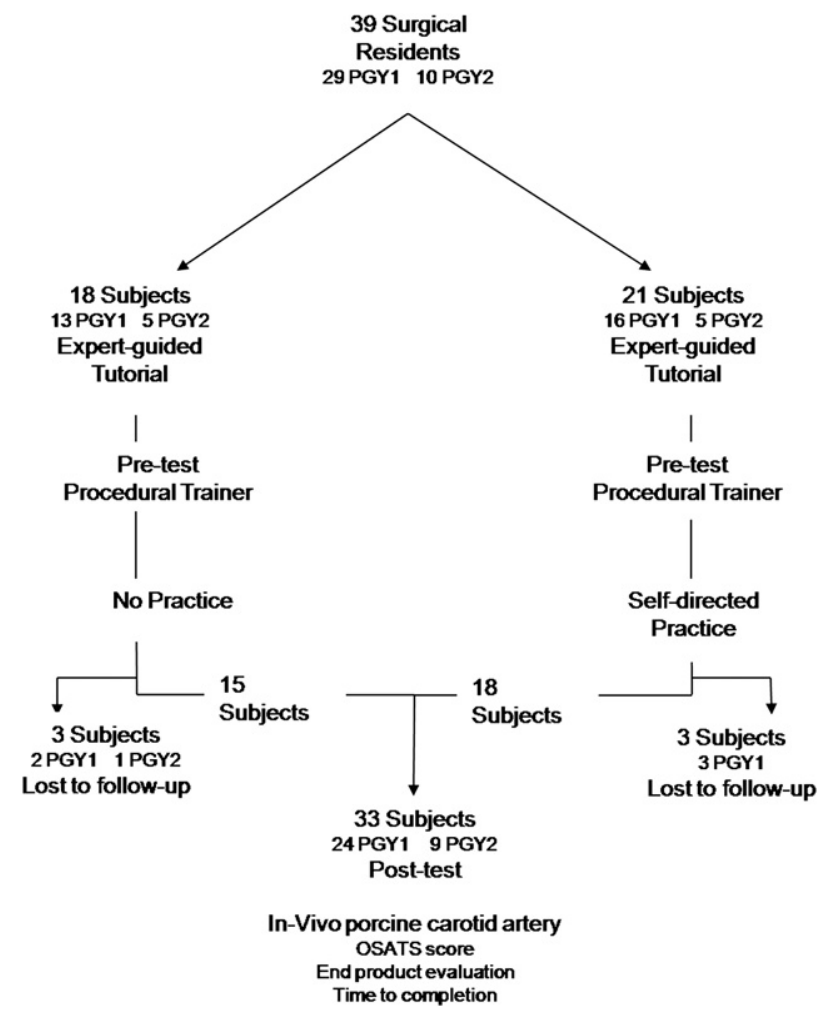

FIGURE 1. CONSORT diagram representing the experimental study design.

NC). A difference of 5 points on the OSATS scale represents a minimal relevant difference between groups. This effect size has been previously demonstrated in a number of simulation trials. ${ }^{3,16}$ It was determined that 34 students, 17 per arm, would provide $80 \%$ power to detect a 5-point difference in mean OSATS score for the in vivo model, assuming a standard deviation of 5.0 and a 2 -sided type 1 error of $5 \%$.

Statistical analysis was performed using SAS version 9.1.3 statistical software (SAS Institute). Demographic data were compared between the groups using a $t$ test for continuous variables and $\chi^{2}$ or Fisher's exact test, where appropriate, for categorical variables. The mean time to completion, mean OSATS scores, and mean end-product scores were compared between groups. The nonparametric Kruskal-Wallis test was used to test for differences between groups in the continuous outcomes. Interrater reliability between the expert observers was calculated using the intraclass correlation coefficient. Given the high interrater reliability, the results of the senior observer were used for the purposes of analysis. Analysis was performed according to the intention-to-treat principle.

\section{RESULTS}

The subject characteristics and pretest scores are shown in Table 1. The distribution of PGY 1 and PGY 2 trainees and the distribution of surgical subspecialties was similar between the randomized groups. Both groups scored similarly on the pretest anastomosis in terms of OSATS scores, end-product scores, and the time to completion. There were no crossovers between randomized groups. All trainees assigned to self-directed practice completed all 10 anastomoses.

Compared with residents who received expert-guided simulator training alone, those who in addition practiced on a simulator independently scored significantly higher on the OSATS scale $(23.7 \pm 4.7$ vs $18.5 \pm 3.9 ; P=.003)$ (Figure 3). When analyzed separately, PGY 1 trainees randomized to self-guided practice scored significantly higher than controls on the OSATS scale $(26.0 \pm 4.6$ vs $20.0 \pm 1.2$; $P=.01)$. PGY 2 trainees also scored significantly higher in technical performance with self-guided practice $(22.8 \pm 4.6$ vs $18.0 \pm 4.4 ; P=.05)$. When the individual component domains of the OSATS scale were tested separately, a statistically significant improvement was observed with independent practice in 4 of the 7 domains. These included time and motion $(P=.005)$, flow of operation $(P=.008)$,

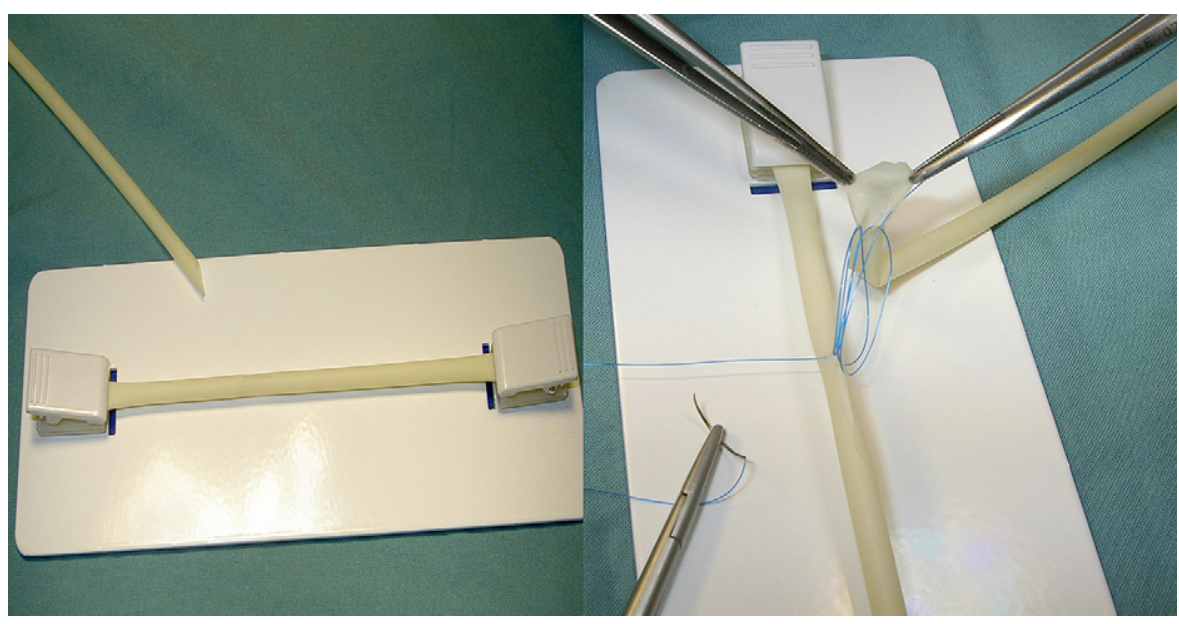

FIGURE 2. Procedural trainer used to simulate an end-to-side anastomosis (Limbs \& Things, Savannah, Ga). 
TABLE 1. Subject characteristics

\begin{tabular}{lccc}
\hline & $\begin{array}{c}\text { Self-directed } \\
\text { practice }(\mathbf{n}=\mathbf{2 1})\end{array}$ & $\begin{array}{c}\text { No self-directed } \\
\text { practice }(\mathbf{n}=\mathbf{1 8})\end{array}$ & $\begin{array}{c}\boldsymbol{P} \\
\text { value }\end{array}$ \\
\hline PGY 1 & $16(76.2 \%)$ & $13(72.2 \%)$ & 1.0 \\
Subspecialty & & & .1 \\
$\quad$ Orthopedic surgery & $3(14.3 \%)$ & $2(11.1 \%)$ & \\
Urology & $3(14.3 \%)$ & $4(22.2 \%)$ & \\
General surgery & $10(47.6 \%)$ & $3(16.7 \%)$ & \\
Cardiac surgery & $1(4.8 \%)$ & $0(0.0 \%)$ & \\
Obstetrics and & $0(0.0 \%)$ & $4(22.2 \%)$ & \\
$\quad$ gynecology & & & \\
Otolaryngology & $1(4.8 \%)$ & $3(16.6 \%)$ & \\
Neurosurgery & $3(14.3 \%)$ & $1(5.6 \%)$ & \\
Plastic surgery & $0(0.0 \%)$ & $1(5.6 \%)$ & \\
Pretest scores & & & .5 \\
OSATS & $20.1( \pm 4.0)$ & $20.0( \pm 4.2)$ & .5 \\
Time to completion $(\mathrm{s})$ & $715( \pm 196)$ & $678( \pm 128)$ & \\
End-product score & $10.7( \pm 2.9)$ & $10.0( \pm 2.7)$ & .8 \\
\hline OSATS, Objective Structured Assessment of Technical Skill.
\end{tabular}

use of assistants $(P=.008)$, and knowledge of specific procedure $(P=.001)$

Residents who practiced independently also scored significantly higher on the end-product evaluation (11.4 \pm 3.2 vs $8.9 \pm 2.1 ; P=.02$ ) (Figure 3 ). When analyzed separately, trainees had nonsignificant trends toward higher OSATS scores with self-guided practice at both the PGY $1(10.7 \pm 3.3$ vs $8.5 \pm 1.9 ; P=.08)$ and PGY $2(13.0 \pm$ 2.5 vs $10.0 \pm 2.3 ; P=.08)$ levels. When the individual component domains of the end-product scale were tested separately, a statistically significant improvement was observed with independent practice in 3 of the 4 domains. These included suture spacing $(P=.04)$, suture eversion $(P=.04)$, and quality of the anastomotic toe $(P=.05)$. In the fourth domain, quality of the anastomotic heel, trainees who practiced independently displayed a nonsignificant trend toward improvement $(P=.09)$.

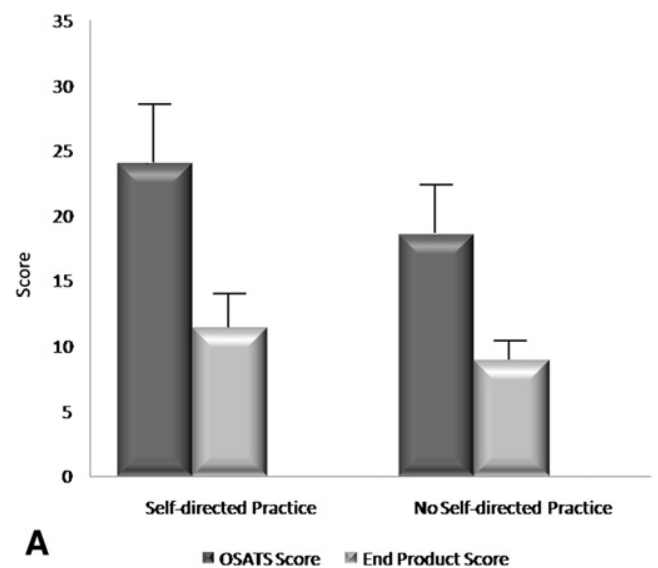

FIGURE 3. The effect of independent, self-directed practice on (A) the total Objective Structured Assessment of Technical Skill (OSATS) score and the total end-product score and (B) the time to completion of the anastomosis on the live porcine carotid artery model.

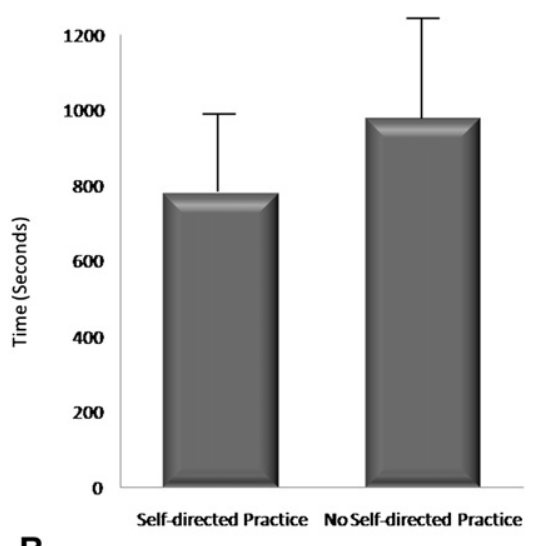

Trainees randomized to self-directed practice on a procedural trainer performed the anastomosis significantly faster (777 seconds vs 977 seconds; $P=.04$ ) (Figure 3 ). The correlation between the 2 expert observers was formally tested. Interrater reliability was high between the expert observers (intraclass correlation coefficient $=0.8$ ).

\section{DISCUSSION}

Teaching surgical technique in a simulated environment has a number of advantages over operating on live patients. Simulation provides the learner with an opportunity for repetitive and deliberate practice of technical skills in a lowconsequence environment. According to Ericsson's theory of acquisition of expert performance, ${ }^{17}$ deliberate practice of skills is the critical activity in developing expert abilities. The intention of the control intervention, the expert-guided tutorial, was to mirror the current reality of surgical training where teaching occurs in the operating room or on a simulator. The purpose of the trial was to assess the possible benefit of additional independent practice in a simulated environment in an actual in vivo operating room scenario. Although after-hours access to procedural trainers has been advocated for trainees, ${ }^{7,18}$ a formal randomized controlled assessment of the value of independent, self-directed simulator training has not been previously conducted.

The findings of the current study suggest the addition of self-directed, after-hours practice on a procedural trainer to an expert-guided tutorial leads to improved technical performance, faster performance, and an improved end product for vascular anastomoses. In addition, it was demonstrated that independent practice on the low-fidelity model does translate into improved performance in a realistic operating room environment. The findings were consistent within the procedural and end-product measures. Furthermore, the findings were consistent at both the PGY 1 and PGY 2 levels individually and within the majority of domains of the

B 
performance scales. These subgroup analyses support the validity and robustness of the overall findings.

The challenges to current methodologies of surgical training underscore the importance of objectively assessing the competence of graduates of surgical residencies. In response to these concerns, surgical training programs have endeavored to develop standardized, objective methods to assess technical skills in trainees. A number of systems have been created and validated as accurate assessments of surgical skill. The University of Toronto OSATS score is the most widely used and validated measure of technical skill. $^{2,10,11,13-15}$ Both the OSATS and time-to-completion are considered measures of generic surgical skill. It has been argued that these techniques ignore the quality of the final surgical result, which represents the more clinically relevant end point. There is growing interest in the use of methodologies that score the quality of the final product. A number of different systems have been specifically developed for the assessment of the quality of an anastomosis. These include Likert scales, which grade the quality of the end product as assessed by experts, ${ }^{2-4,12}$ maximal diameter of the anastomosis, ${ }^{12}$ a dichotomous assessment of patency, ${ }^{3,4}$ and assessing the degree of leak using a Likert scale. ${ }^{9}$ To ensure a robust assessment of outcomes, we used 2 procedural outcome measures and an assessment of the end product, specifically the anastomosis-specific 4-parameter global rating scale by blinded observers. ${ }^{12}$

A number of studies have demonstrated the utility of simulation training on the performance of various types of anastomoses. Grober and associates ${ }^{3}$ demonstrated the effectiveness of training on high-fidelity and low-fidelity models for the performance of end-to-end microanastomosis on the vas deferens of a live rat. Simulator training, regardless of model fidelity, was found to improve subsequent performance of the task on both high- and low-fidelity simulators compared with subjects who received didactic training alone. This benefit was found to be durable and persist in the long-term. ${ }^{4}$ Pandey and colleagues ${ }^{2}$ examined the effect of participation in a simulator-based workshop on the performance of an end-to-end anastomosis for abdominal aortic aneurysm repair. The authors demonstrated objective improvements in technical performance for surgeons of various levels of experience after participation in the workshop. Reporting the experience from the Thoracic Surgery Directors Association "Boot Camp" experience, Fann and coworkers ${ }^{8}$ reported improved performance of coronary anastomosis after an expert-guided tutorial on a task trainer and porcine heart model. This trial confirms the findings of Fann and coworkers and contributes novel information about the value of after-hours, self-directed practice.

The concept of self-directed practice on a procedural trainer during nonclinical time has been examined in cardiac surgery. The combination of formative feedback from an expert combined with 2 weeks of self-directed independent practice on a low-fidelity procedural trainer resulted in improved technical performance of mitral valve annuloplasty by cardiac surgical trainees at multiple levels. ${ }^{18}$ In a study examining the role of simulation in coronary anastomosis, Fann and coworkers ${ }^{7}$ examined the effect of a simulator training protocol, which consisted of both expert-guided and self-directed practice. Although not a randomized comparison, the authors demonstrated that this training regimen resulted in improved performance on a procedural trainer for arrested-heart coronary anastomosis and on a high-fidelity simulator for beating-heart coronary anastomosis. The authors concluded that distributed practice, including self-directed practice, is a useful method for training surgical skills. In our study, we have confirmed the importance of self-directed practice during the trainee's nonclinical time. Fann and colleagues noted that improvement was not observed in all residents and that there is likely a ceiling effect with respect to the trainer and a plateau effect with respect to the trainee. The authors noted that this was more likely the case in more senior trainees with greater experience. This observation has been made previously. It has been demonstrated that, for junior trainees, model fidelity does not affect the degree of benefit conferred by training. ${ }^{3,4,16}$ As surgical experience increases, the fidelity of the model contributes to the ceiling effect. In the current study, junior surgical trainees with limited exposure to cardiovascular surgery were selected to limit these effects.

The results of this study have important implications for cardiovascular surgical training. As challenges to traditional methods of teaching surgical skills in the operating room continue to increase, the importance of learning skills in a simulated environment will gain increasing importance. As such, simulation has become an important component of surgical curricula. Of particular importance is the unknown effect of ever-increasing work-hour restrictions for trainees on the quality of technical surgical training. Our findings suggest that providing after-hours access for trainees to procedural simulators is an effective technique to improve technical skill in the operating room environment. As such, trainees can effectively practice procedural skills on their own time to tangibly improve technical performance. This approach has the added benefits of maximizing usage of work hours for work-related tasks and optimizing the trainee's experience while in the operating room.

Several authors have expressed concern over the feasibility and sustainability of simulation training, particularly high-fidelity simulation, because of cost, time, and complexity. ${ }^{19,20}$ The procedural trainer used in the current trial has the benefits of being inexpensive, lowmaintenance, and portable. These characteristics are critical in developing a system for simulation training, including self-directed practice, which is sustainable and feasible for both the training program and the trainee. 


\section{LIMITATIONS}

Although the trainees randomized in this study were well matched in terms of level of training and performance on the anastomosis pretest, only junior trainees in the first 2 years of surgical training were included. The results observed may not be generalizable to trainees of higher levels of experience. We used a live porcine carotid artery model in an operating room environment to realistically simulate a live human patient. Despite this, it is difficult to entirely simulate the complex setup of a coronary anastomosis. Furthermore, the inability to suspend disbelief may have been a factor for some trainees using the porcine model. Finally, subjects randomized to self-directed practice self-reported the anastomoses performed independently. As such, the potential for misclassification is always a possibility and the level of intensity of independent practice may have varied.

\section{CONCLUSIONS}

Residents who had the opportunity for self-directed simulator practice performed an end-to-side anastomosis more adeptly, more quickly, and with a higher quality end product. The results of this randomized trial suggest that independent training on a procedural trainer did transfer to improved performance in an operating room environment. Simulator training should be incorporated into cardiovascular surgical curricula, and residents should have access to this modality for independent practice to improve operating room performance.

\section{References}

1. Reznick RK, MacRae H. Teaching surgical skills—changes in the wind. $N$ Engl J Med. 2006;355:2664-9.

2. Pandey VA, Black SA, Lazaris AM, Allenberg JR, Eckstein HH, Hagmuller GW, et al. Do workshops improve the technical skill of vascular surgical trainees? Eur J Vasc Endovasc Surg. 2005;30:441-7.

3. Grober ED, Hamstra SJ, Wanzel KR, Reznick RK, Matsumoto ED, Sidhu RS, et al. The educational impact of bench model fidelity on the acquisition of technical skill: the use of clinically relevant outcome measures. Ann Surg. 2004;240:374-81.

4. Grober ED, Hamstra SJ, Wanzel KR, Reznick RK, Matsumoto ED, Sidhu RS, et al. Laboratory based training in urological microsurgery with bench model simulators: a randomized controlled trial evaluating the durability of technical skill. J Urol. 2004;172:378-81

5. Anastakis DJ, Regehr G, Reznick RK, Cusimano M, Murnaghan J, Brown M, et al. Assessment of technical skills transfer from the bench training model to the human model. Am J Surg. 1999;177:167-70.

6. Seymour NE, Gallagher AG, Roman SA, O'Brien MK, Bansal VK, Andersen DK, et al. Virtual reality training improves operating room performance: results of a randomized, double-blinded study. Ann Surg. 2002;236:458-63.

7. Fann JI, Caffarelli AD, Georgette G, Howard SK, Gaba DM, Youngblood P, et al. Improvement in coronary anastomosis with cardiac surgery simulation. J Thorac Cardiovasc Surg. 2008;136:1486-91.

8. Fann JI, Calhoon JH, Carpenter AJ, Merrill WH, Brown JW, Poston RS, et al. Simulation in coronary artery anastomosis early in cardiothoracic surgical residency training: the Boot Camp experience. J Thorac Cardiovasc Surg. 2010;139: 1275-81.

9. Wilasrusmee C, Phromsopha N, Lertsitichai P, Kittur DS. A new vascular anastomosis model: relation between outcome and experience. Eur J Vasc Endovasc Surg. 2007;33:208-13.

10. Hance J, Aggarwal R, Stanbridge R, Blauth C, Munz Y, Darzi A, et al. Objective assessment of technical skills in cardiac surgery. Eur J Cardiothorac Surg. 2005; 28:157-62.
11. Pandey VA, Wolfe JH, Lindahl AK, Rauwerda JA, Bergqvist D. Validity of an exam assessment in surgical skill: EBSQ-VASC pilot study. Eur J Vasc Endovasc Surg. 2004;27:341-8.

12. Datta V, Mandalia M, Mackay S, Chang A, Cheshire N, Darzi A. Relationship between skill and outcome in the laboratory-based model. Surgery. 2002;131: 318-23.

13. Faulkner H, Regehr G, Martin J, Reznick R. Validation of an objective structured assessment of technical skill for surgical residents. Acad Med. 1996;71:1363-5.

14. Martin JA, Regehr G, Reznick R, MacRae H, Murnaghan J, Hutchison C, et al Objective structured assessment of technical skill (OSATS) for surgical residents. Br J Surg. 1997;84:273-8.

15. Reznick R, Regehr G, MacRae H, Martin J, McCulloch W. Testing technical skil via an innovative "bench station" examination. Am J Surg. 1997;173:226-30.

16. Sidhu RS, Park J, Brydges R, MacRae HM, Dubrowski A. Laboratory-based vascular anastomosis training: a randomized controlled trial evaluating the effects of bench model fidelity and level of training on skill acquisition. J Vasc Surg. 2007 45:343-9.

17. Ericsson KA. Deliberate practice and the acquisition and maintenance of expert performance in medicine and related domains. Acad Med. 2004 Oct;79(10 Suppl):S70-81.

18. Joyce DL, Dhillon TS, Caffarelli AD, Joyce DD, Tsirigotis DN, Burdon TA, et al. Simulation and skills training in mitral valve surgery. J Thorac Cardiovasc Surg. 2011;141:107-12.

19. Feins RH. Expert commentary: cardiothoracic surgical simulation. J Thorac Car diovasc Surg. 2008;135:485-6.

20. Ramphal PS, Coore DN, Craven MP, Forbes NF, Newman SM, Coye AA, et al. A high fidelity tissue-based cardiac surgical simulator. Eur J Cardiothorac Surg. 2005;27:910-6.

\section{Discussion}

Dr W. Randolph Chitwood, Jr (Greenville, $N C$ ). This is a very interesting study. Many of us believe that more training is better and more simulation training will be better premonitory training. As an example, I trained for 10 years, and future residents now may be training for 6 years. Moreover, residents now have limited work hours; therefore, they may obtain only about one third to one half of the actual experience that past surgeons had in residency training. Young surgeons will have to make up this difference in education with something else.

How will you take this platform to the next level as a higherfidelity training program? Today, there are simulators that will allow young surgeons to do vascular anastomoses. How does your work compare?

Dr Price. Thank you, Dr Chitwood, you have raised a number of interesting points. Your first point, with which I entirely agree, is that the shorter duration of training combined with the challenges I mentioned certainly contribute to decreased opportunity to train technical skills. The issue of model fidelity is an important one, and there has actually been quite a bit of literature in education scholarship looking at that. What has been found is that high model fidelity is important in more advanced surgical trainees. In lowerlevel trainees, specifically PGY 1 and PGY 2 trainees, which we examined in this study, model fidelity seems to be less of an issue. That is to say that junior trainees benefit a great deal from a lowfidelity model. A low-fidelity model has the additional benefit of making a simulation program feasible and cost effective.

To answer your question regarding how this can be taken forward combining low- and high-fidelity models, I think that resources have to be focused in terms of cost at the level of trainee. For lower-level trainees, I think low-fidelity models are very effective and cost effective as well. For higher-level trainees who will want to benefit from simulation training, perhaps higher-fidelity 
models should fill that role. Finally, I would like to add that simulation training for a junior trainee increases the efficiency of clinical time for the residents; they benefit more from their time in the operating room because they come in with a higher level of baseline skills.

Dr M. Blair Marshall (Washington, DC). That was a very nice study. I have 2 questions. One, did you notice a difference in the residents' interest in practicing their skills independently?

Dr Price. I'm sorry. Are you asking whether I noticed a difference between the groups?

Dr Marshall. The residents who were relegated to practicing on their own, did some practice more than others?

Dr Price. The amount of practice was actually protocol driven. We wanted to avoid variable amounts of practice as a source of bias. It was dictated by the protocol that those randomized to independent practice performed exactly 10 anastomoses on their own time, and they actually logged this in a logbook. The subjects were instructed not to do more and not to do less.

Dr Marshall. Did you have any difficulty getting that done with them?
Dr Price. By resident report, all completed all 10 anastomoses on their own time, and they submitted the logbook to us for evaluation.

Dr Marshall. Second, your tendency is to demonstrate differences in a technical skill using a simulated environment that replicates an operating room. However, there have been studies showing that one's ability to determine differences in technical skills acquired is worse than if they are tested in a less stressful environment. What are your thoughts on that?

Dr Price. You have raised an interesting point. The reason we designed the trial in this way is that we believed the most relevant outcome was that practicing on their own on a simulator would improve their performance when they came to the operating room. The intention was to design as realistic a high-fidelity model as possible on which to assess the primary outcome. Therefore, it was a live model. We adhered to operating room protocol in terms of sterility, monitors, and intubation, and a pseudo-anesthetist was present. All these factors were meant to actually add to the stress of the situation and realistically simulate the actual operating room environment. We considered that the most relevant way to judge performance.

APPENDIX 1. Objective Structured Assessment of Technical Skill (OSATS) global rating scale

Respect for tissue

Time and motion

Instrument handling

Knowledge of instruments

Flow of operation

Use of assistants

Knowledge of specific procedure

2

Frequently used unnecessary force on tissues and or caused damage by inappropriate use of instruments

$$
1
$$

Many unnecessary moves

Repeatedly made tentative or awkward moves with instruments by inappropriate use of instruments

Frequently asked for wrong instrument or used inappropriate instrument

Frequently stopped operating and seemed unsure of next move

Consistently placed assistants poorly or failed to use assistants

Deficient knowledge. Needed specific instruction at most steps

Careful handling of tissue but occasionally caused inadvertent damage

2

Efficient time/motion, but some unnecessary moves

2

Competent use of instruments but occasionally appeared stiff or awkward

Knew names of most instrument and used appropriate instrument operation

Demonstrated some forward planning with reasonable progression of procedure

$$
3
$$
of the time$$
3
$$

$4 \quad 5$

Consistently handled tissues appropriately with minimal damage

4 5

Clear economy of movement and maximum efficiency

5

Fluid moves with instruments and no awkward moves

4

Obviously familiar with the instruments and their names

Appropriate use of assistants most

Knew all important steps of the
4

5

Obviously planned course of operation with effortless flow from one move to the next

Strategically used assistants to the best advantage at all times

Demonstrated familiarity with all steps of the operation 
APPENDIX 2.

Subject study No.:

Lab date (dry/wet):

Judge:

Date of assessment:

End-product rating scale

Suture spacing

1

2

3

Bites inconsistent in size and depth, with wide gaps

Suture eversion

$$
\text { Inadequate }
$$

$$
1
$$

3

Quality of anastomotic heel

Inadequate

Quality of anastomotic toe

Inadequate

2

2

1

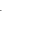

.

Total score: /20

Notes: 